\title{
Same Segment Early Recurrence in Surgery of Lumbar Canal Stenosis- Role of Dissectomy
}

\author{
Dr. Amit Agarwal ${ }^{1}$, Dr. Imran Sajid ${ }^{2}$, Dr. Namit Singhal ${ }^{3}$, Dr. Anubhav \\ Agarwal $^{4}$ \\ \& Dr. Harish Chandra ${ }^{5}$ \\ ${ }^{1}$ Associate Professor, M.S. (Ortho), M.Ch. (Ortho), F.H. Medical Colllege, Agra. \\ ${ }^{2}$ Assistant Professor, M.S. (Ortho), M.Ch. (Ortho), F.H. Medical Colllege, Agra. \\ ${ }^{3}$ M.S., M.Ch., Neuro Surgeon, S.S. Hospital, Agra \\ ${ }^{4}$ M.S., M.Ch. (Ortho), Chandra Orthopedic \& Maternity Institute, Agra \\ ${ }^{5}$ M.S.(Ortho), Chandra Orthopedic \& Maternity Institute, Agra
}

\begin{abstract}
:
Background: Symptomatic lumbar canal stenosis (LCS) usually responds well to surgery. However, in a subset of patients symptoms recur after variable periods of time.

Aims: The aim of this study was to identify subset of patients having same segment early recurrence (recurrence $<2$ years) in surgery of LCS and the role of dissectomy in causing it.

Materials And Methods: A prospective analysis of 100 patients who were operated for LCS from 2013 to 2015. All patients were followed up for atleast 2 years.

Inclusion Criteria: Symptomatic LCS with or without Grade 1 fixed listhesis.

Exclusion Criteria: LCS associated with Listhesis of grade 2 or more, LCS with mobile listhesis of any grade, presence of degenerative scoliosis and follow up more than 2 years. Based on type of surgical procedure performed initially patients were divided into 3 categories. CATEGORY-1 Decompression via laminectomy and foraminotomy. CATEGORY-2 Category 1 along with dissectomy. CATEGORY-3 Category 2 with TLIF combined. Redo surgical options included 1. Excision of scar and foraminotomy. 2. Excision of scar and pedicle screw rod fixation. 3. Only pedicle screw rod fixation with distraction.

Results: In category 1 out of 5 patients (9.09\%) developed recurrence, in category 2 out of 9 patients (22.5\%) developed recurrence ( $p<0.0001)$.

Conclusions: The strongest predictor of early recurrence is dissectomy combined with decompression. Excision of disc material leads to vertebral settling resulting in loss of height of foraminal space. So in surgery for LCS, dissectomy should only be done if frank herniation is present causing significant compression. When dissectomy is done it should always be supplemented with stand alone TLIF. In redosurgery, excision of scar tissue leads to major dural tears. Pedicle screw rod fixation with distraction is an excellent option in these cases as it leads to restoration of foraminal height.
\end{abstract}

\section{Introduction}

Symptomatic lumbar canal stenosis usually responds well to surgery. Patients of lumbar canal stenosis usually present with neurogenic claudication, occasionally with radicular pain. Radicular Pain in this subset of patients usually gets relieved on sitting, in contradiction to radicular pain of patients with disc prolapse. This contradiction is due to compression being dorsal to neural structures in LCS. Sometimes patients ofLCS have associated with spinal instability.Traditionally spinal instability is defined as "loss of ability of spine under physiological loads to maintain relationships between vertebrae in such a way that there is neither damage nor subsequent irritation to spinal cord or nerve roots and in addition, there is no development of incapacitating deformity or pain due to structural changes". Louis two column concept assigns significance to vertebral body and facet joint complexes on either side of spine. Dennis emphasizes on three column concept: spinal instability is said to be present when any two columns are involved. Hence according to Dennis concept, grade1 fixed spondylolisthesis is stable; rest others are unstable. Preoperative identification of instability is very important as this leads to high chances of early recurrence. There are only few studies who have dealt with recurrence of symptoms, not responding to conservative measures, after decompressive surgery for LCS. This studyaims to identify subset of patients of LCS who have a higher change of early recurrence ( $<2$ years), using the JOA score. We have also analyzed the surgical options in redo surgery. 


\section{Materials And Methods}

This prospective study was conducted at our hospital fromJanuary 2013 to July 2015. During this period, 100 patients of degenerative lumbar canal stenosis were operated for decompressive surgery based on the inclusion and exclusion criteria. Patients who had significant claudication distance with or without posturerelated radicular pain were offered surgery. Significant claudication was defined as the claudication distance which interferes with carrying out of their routine daily activities. The exclusion Criteria included the following:LCS associated with spondylolisthesis of grade 2 or more, mobile spondylolisthesis of any grade, presence of degenerative scoliosis, presence of spondolysis defect, presence of pars defect and follow up after 2 years.Medial facetectomy more than $1 / 3$ during index surgery and recurrence associated with listhesis of any grade. All these patients were evaluated with dynamic lumbar x-rays and MRI scans. Radiological parameters which were assessed included the following: Central canal dimensions in saggital plane, Lateral recess dimensions in saggital plane, presence of PIVD causing significant compression, presence of spondylolisthesis, presence of degenerative scoliosis and presence of spondolysis defect.

On basis of first surgical procedure performed, patients were grouped in three categories.

- Category-1: decompression via laminectomy and foraminotomy

- Category-2: decompression via laminectomy and foraminotomy along with dissectomy.

- Category-3: decompression via laminectomy and foraminotomy along with dissectomy supplemented with stand alone Transforaminal lumbar Interbody fusion (TLIF).

All procedures were performed by senior orthopedic surgeon. According to this protocol, 55 cases were operated in category 1 and 40 patients were operated in category 2 . In 5 cases, dissectomy with foraminotomy was supplemented with stand alone TLIF (Fig 2). Average follow-up period was 9.6 months (range: 3-24 months). Recurrence was divided as early recurrence defined as recurrence of same symptoms in less than 2 years. Patients having recurrence beyond 2 years were excluded from the study. Pre and post recurrent symptoms were assessed according to JOA evaluation system for low back pain. The JOA score was determined by direct questioning to assess subjective symptoms, clinical signs, and restriction of activities of daily living. 14 Patients who developed recurrence were subjected to either of the following procedures:

- Excision of scar and foraminotomy

- Excision of scar and pedicle screw fixation

- Only pedicle screw fixation with distraction to prevent dural tears

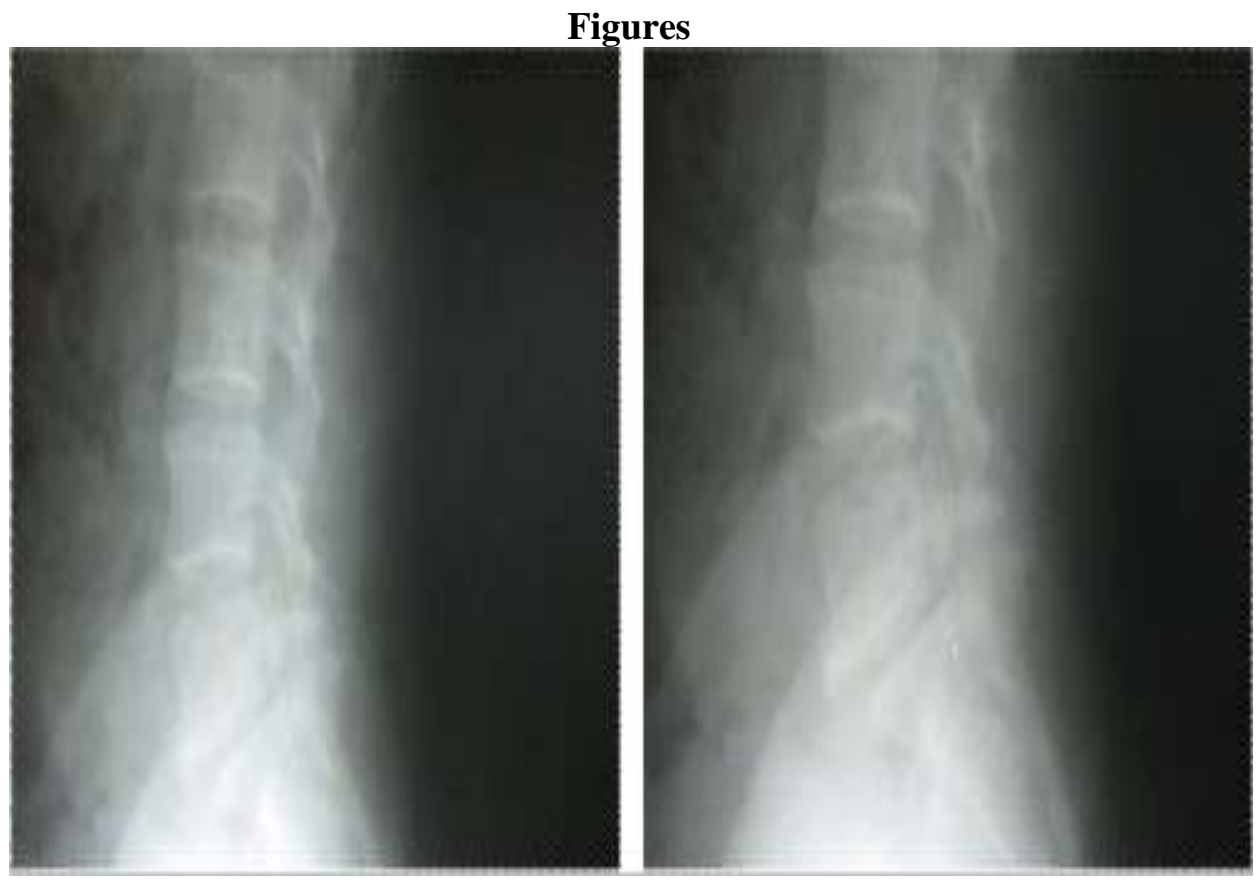

Fig1a. Preoperative dynamic X-rays of a 60years old male patient who presented with degenerative LCS at L4L5, L5-S1 with grade 1 fixed listhesis of L5 over S1. His preoperative JOA score was 5. Grade 1 fixed listhesis never predisposes to instability. 


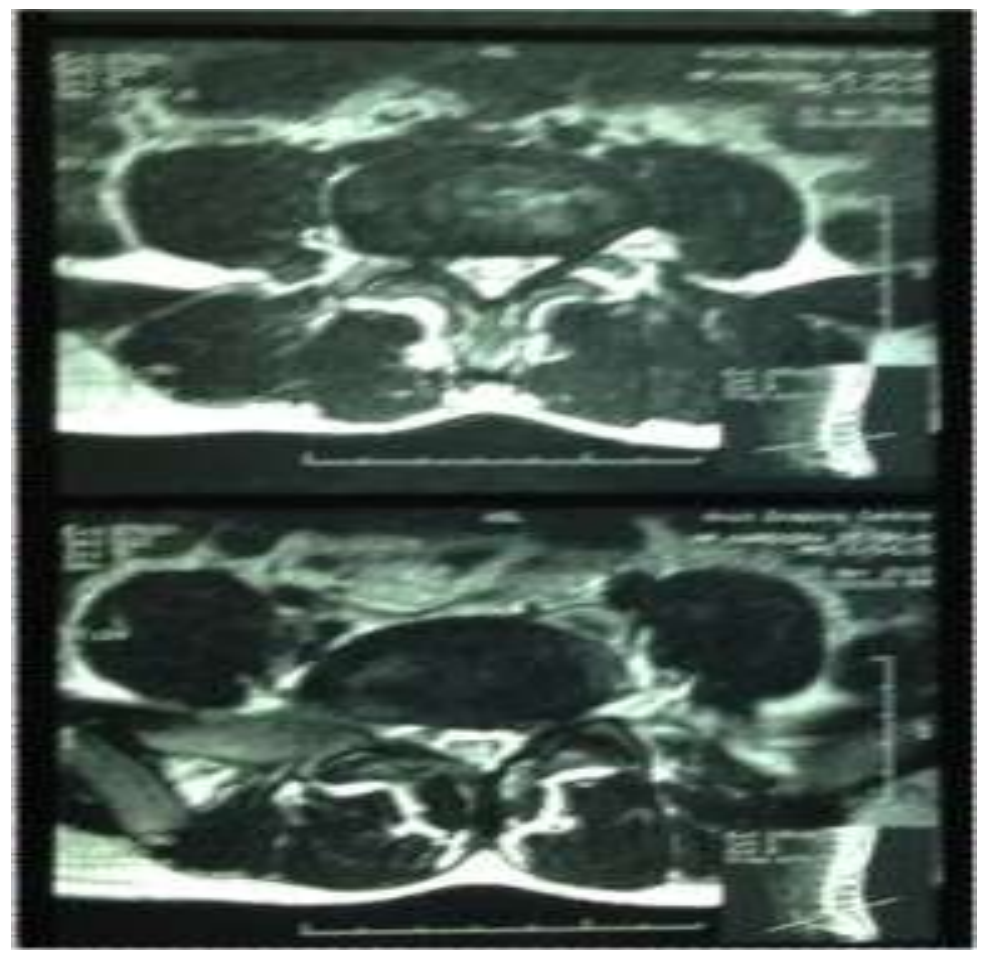

Fig1b.Preoperative T2 saggital MRI section of the same patient showing degenerative LCS L4-5, L5-S1 without any disc herniation.

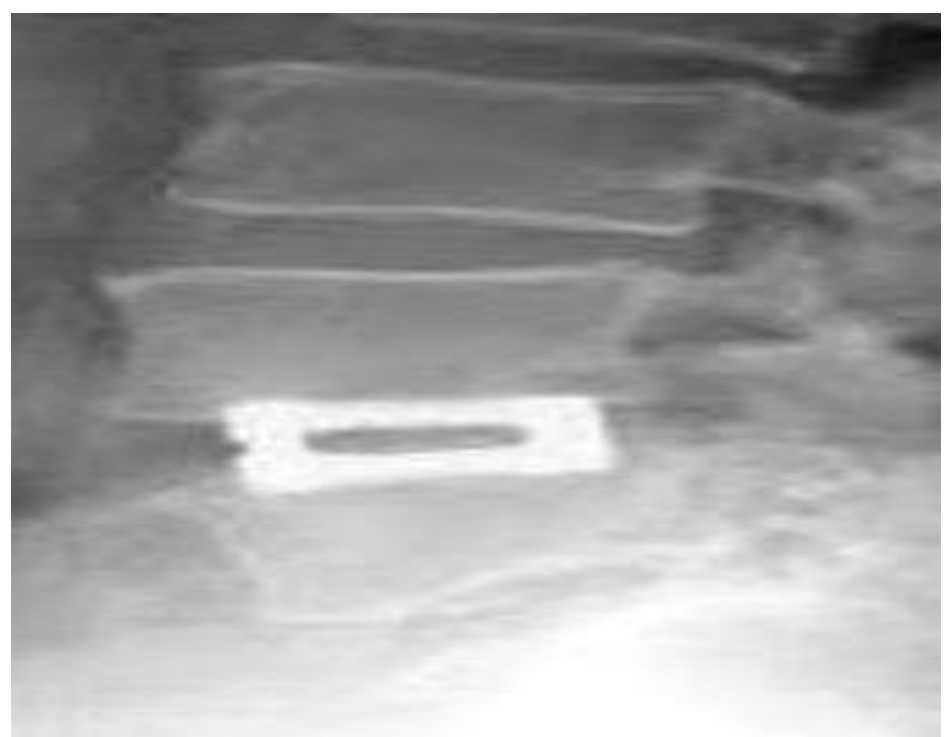

Fig2. Category-3 patient treated with spinal decompression supplemented with stand alone TLIF after dissectomy

\section{Statistical Analysis} data.

JOA scores at recurrence in the categories 1 and 2 were compared using chi square test for parametric

\section{Results}

The average age was 48.5 years (range 27-74 years). There were 72 males and 38 females. All the patients failed to respond to conservative measures for 12 weeks. Out of 55 patients who were operated in category $1,5(9.09 \%)$ developed recurrence. The average duration at which recurrence developed was 15 months 12 days. The average JOA score of thissubset of patients who developed recurrence was 16.6. 40 patients were operated in category 2 in whom dissectomy was also done to achieve neural decompression. Out of 40 patients, $9(22.5 \%)$ developed recurrence. The average duration at which recurrence developed was 12 months 2 days. The average JOA score of these patients who developed recurrence was 10.2

DOI: 10.9790/0853-1508023440 $\quad$ www.iosrjournals.org $\quad 36 \mid$ Page


During later part of the study, in 5 patients who were subjected to additional dissectomy to achieve neural decompression, stand alone TLIF was also done to prevent vertebral settling. None of these patients developed recurrence on a follow up of two years.

14patients who developed recurrence were subjected to the following procedures: Excision of scar and foraminotomy was done in only 2 patients. In 2 patients, excision of scar and pedicle screw fixation was done. Due to presence of significant dural tears in patients with excision of scar, only pedicle screw fixation with distraction was done in 10 patients with significant improvement in all the cases.

On comparison of JOA scores in categories 1 and 2 of patients using chi square test for parametric data, $P$ value is $<0.0001$ which meant that outcomes were extremely significant postoperatively (Table 1$)$.

Table 1-

\begin{tabular}{|l|c|c|}
\hline & Recurrence & Average JOA score at recurrence \\
\hline Category I (55) & 5 & 16.8 \\
\hline Category II (40) & 9 & 10.1 \\
\hline t-value & -9.3021 & 7.6409 \\
\hline p-value & $<0.0001$ & $<0.0001$ \\
\hline
\end{tabular}

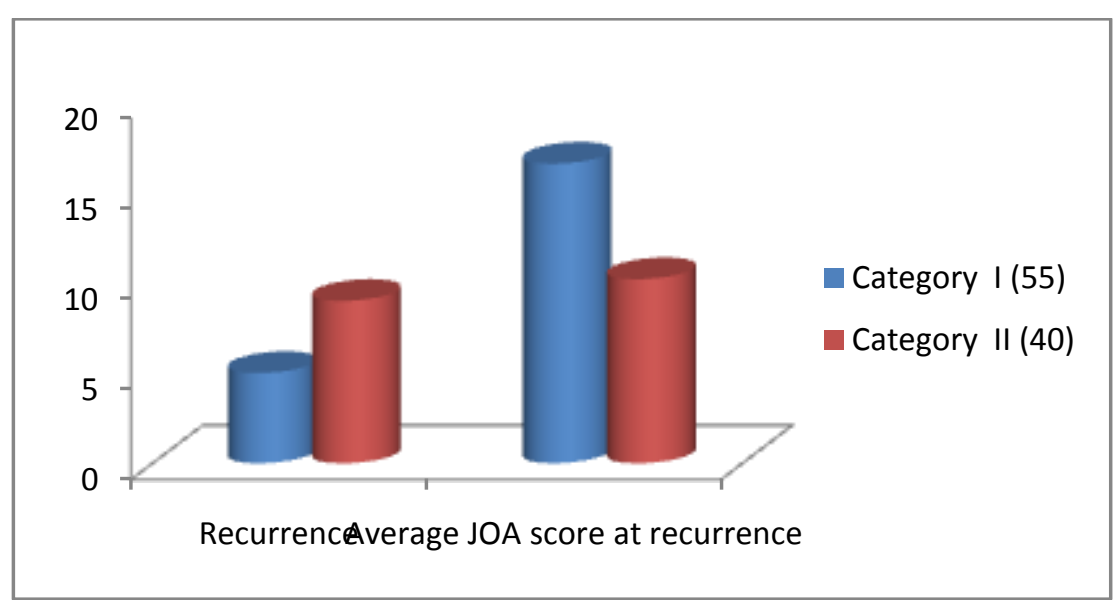

Table-2 - Recurrence rate of Category-1

\begin{tabular}{|c|c|c|c|}
\hline Age & Sex & JOA score & $\begin{array}{c}\text { Duration of } \\
\text { recurrence after } 1^{\text {st }} \\
\text { surgery (months) }\end{array}$ \\
\hline 30 & $\mathrm{M}$ & 21 & 20 \\
\hline 36 & $\mathrm{~F}$ & 20 & 16 \\
\hline 48 & $\mathrm{M}$ & 14 & 14 \\
\hline 56 & $\mathrm{~F}$ & 12 & 12 \\
\hline 65 & $\mathrm{M}$ & 17 & 15 \\
\hline
\end{tabular}

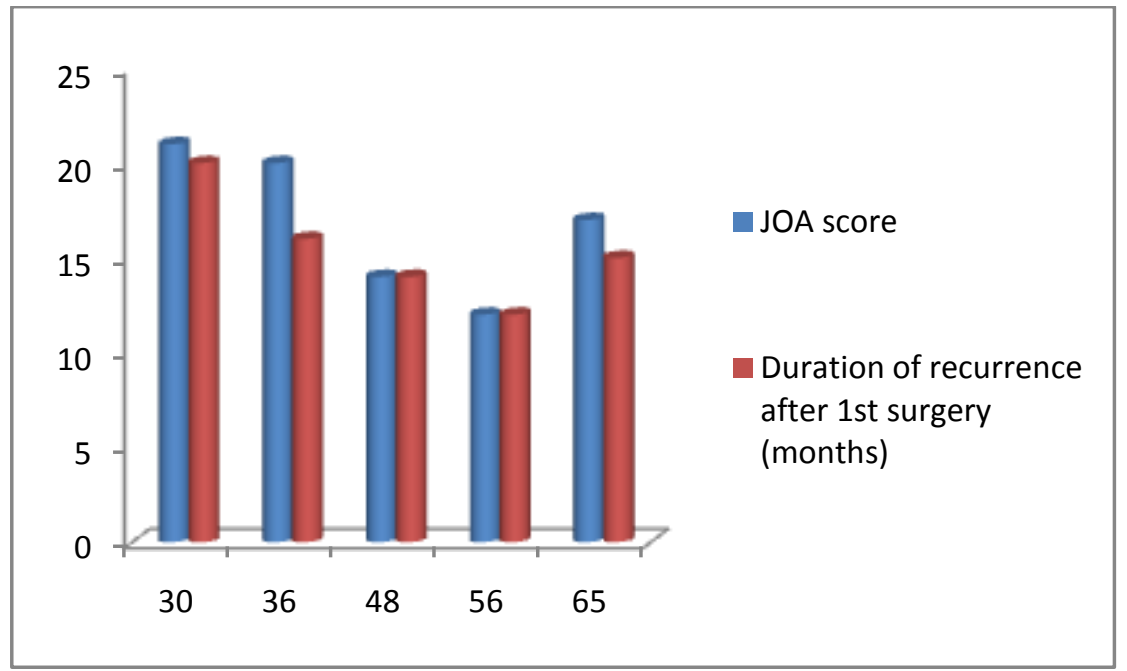


Table-3 - Recurrence rate of Category-2

\begin{tabular}{|c|c|c|c|}
\hline Age & Sex & JOA score & $\begin{array}{c}\text { Duration of } \\
\text { recurrence after } 1^{\text {st }} \\
\text { surgery (months) }\end{array}$ \\
\hline 28 & M & 8 & 14 \\
\hline 34 & F & 12 & 10 \\
\hline 40 & M & 9 & 11 \\
\hline 46 & F & 13 & 12 \\
\hline 50 & M & 11 & 16 \\
\hline 54 & M & 9 & 14 \\
\hline 60 & M & 8 & 12 \\
\hline 62 & F & 12 & 11 \\
\hline 72 & M & 9 & 9 \\
\hline
\end{tabular}

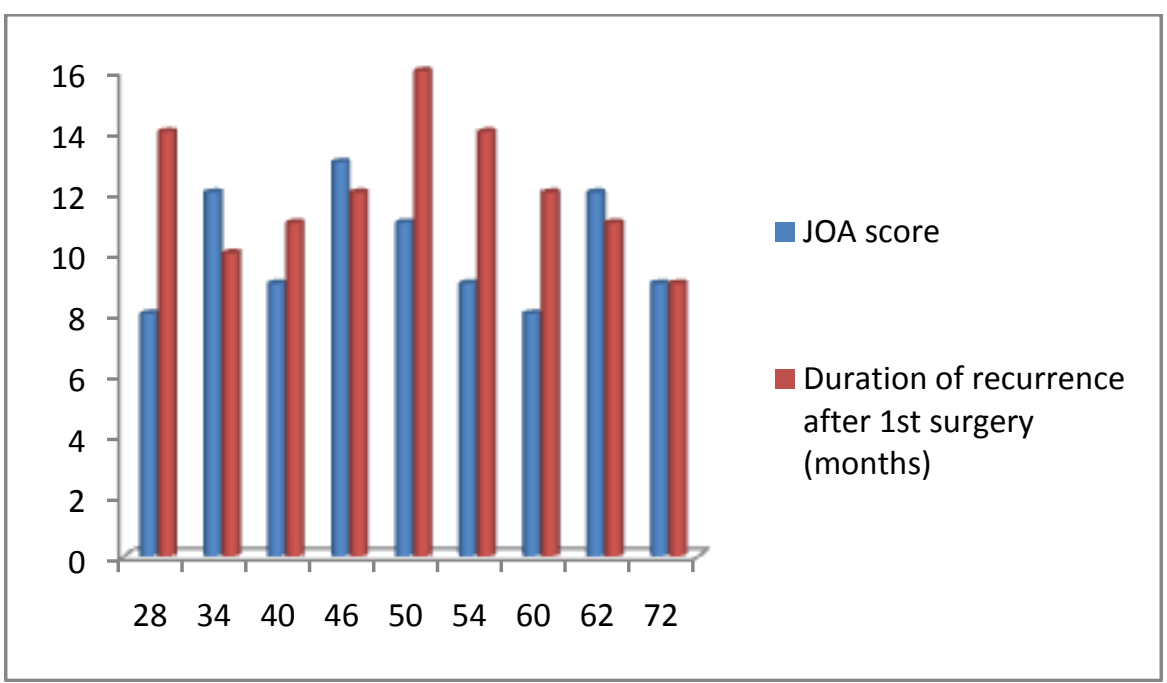

Japanese Orthopedic Association Score (JOA)

Name and Synonyms

Japanese orthopaedic association score, JOA Score.

Structure / Content

(Modified by Keller 1993)

Criterion Points

Motor function

- Paralysis 1

Upper extremity

- $\quad$ Fine motor function massively decreased 2

- $\quad$ Fine motor function decelerated 3

- Discreet weakness in hands or proximal arm 4

- $\quad$ Normal function 5

\section{Motor function}

- Unable to walk 1

\section{Lower extremity}

- Need walking aid on flat floor

- Need handrail on stairs 3

- Able to walk without walking aid, but inadequate

- Normal function 5

\section{Sensory Upper extremity/lower extremity/trunk}

- Apparent sensory loss

- Minimal sensory loss

- Normal function 3 


Bladder function
$\begin{array}{ll}\text { - } & \text { Urinary retention } \\ \text { - } & 1 \\ \text { - } & \text { Severe dysfunction } \\ \text { - } & \text { Mild dysfunction } \\ \text { - Normal function } & 3 \\ \end{array}$

\section{Scoring Method and Interpretetion}

Total score $\quad 0-17$. The lower the score the more severe the deficits.

- Normal function $16+17$

- Grade 1: 12-15

- Grade 2: 8-11

- Grade 3: 0-7

Weight of the criterion in percentage of 17 points: upper extremity $23.5 \%$; lower extremity $23.5 \%$; sensory $3 \times 11.8 \%$ (total: $35.4 \%$ ); bladder and bowel function $17.6 \%$

\section{Discussion}

Surgery for lumbar canal stenosis is commonly performed and generally results in substantial symptom relief. Symptomatic LCS is among the most common indications for lumbar spine surgery. Radiographically, the prevalence of LCS increases with age, being present in 44\% of individuals aged 60-69 years. It implies that the number of operations for LCS will increase in forthcoming years as life expectancy continues to lengthen. Unfortunately, recurrent stenosis develops in many cases, which can lead to increased morbidity as compared to the index surgery. Recurrent stenosis can develop at the index, adjacent, or distant levels of the lumbar spine. Recurrent stenosis can arise from a variety of mechanisms and presents with a broad spectrum of symptoms

There are a number of reports detailing varied reoperation rates follow index surgery for LSS. Javalkar et al reviewed the records of 335 patients undergoing surgical treatment for LSS and found that $13 \%$ required revision surgery at a mean of 16 months after the index operation ${ }^{1}$. The most common indication was recurrent LCS at the previously operated level. Similarly, Juricek et al documented an $8.7 \%$ rate of revision surgery ${ }^{2}$.The incidence of recurrent stenosis is intimately related to the time elapsed from the index procedure. In one study, the average time interval to revision surgery for same level recurrent LSS was 4.16 years ${ }^{3}$. Another study found revision procedures were performed at an average of 35 months after index surgery, while the study by Javalkar, as noted above, reported a mean of 16 months ${ }^{4}$. Atlas et al observed that $23 \%$ of patients underwent revision LSS surgery within 10 years ${ }^{5}$. Recurrence can be divided as early recurrence defined as recurrence of same symptoms in less than 2 years.

Multiple factors are involved in the recurrence of symptoms for LCS. These factors determine whether recurrence is at the same segment or adjacent segment. It has been suggested that the rate of reoperation can be influenced by the type of operation performed initially and other co-variables. One group of investigators noted that the interval between index and revision surgery was shorter in patients with diabetes ${ }^{6}$. Deyo et al found that repeat surgery was less likely with older age at index surgery ${ }^{7}$. The presence of grade I degenerative spondylolisthesis or degenerative scoliosis does not appear to impact the rate of revision LSS surgery. The development of late recurrence, recurrence after 2 years, has been linked to motion patterns and instability. No study has ever evaluated the role of dissectomy in patients of LCS with early recurrence. In one series, postoperative instability was deemed responsible for recurrent symptoms in $25 \%$ of reoperations for lumbar stenosis $^{8}$. Instability can also occur at levels adjacent to a previous fusion and decompression. We have excluded all cases with preoperative instability and those cases which had recurrence associated with instability.

In this study, we aim to find the role of dissectomy in leading to same segment early recurrence in patients of LCS. On comparison of JOA scores in categories 1 and 2 of patients using chi square test for parametric data, $P$ value is $<0.001$ which means that dissectomy is an important predictor of early recurrence. Excision of disc material leads to vertebral settling resulting in loss of height of foraminal space. This predisposes to recurrence. Interestingly, recent research has shown that intervertebral height loss leads tothickening and hypertrophy of the LF due to buckling and redundancy ${ }^{9}$. This was exemplified by addition of TLIF after dissectomy in 5 patients, whereby it prevented vertebral body settling and hence recurrence.

In those cases that are recalcitrant to nonoperative treatment, revision decompression surgery may be elected, sometimes involving fusion and/or stabilization. Results of revision surgery for recurrent LSS can be difficult to interpret, success rates have ranged widely from $12 \%$ to $82 \%$. In one cohort study of elderly patients undergoing non-fusion procedures, only $36 \%$ were found to be "very" or "somewhat satisfied.Chen et al. showed good or excellent clinical results in nearly $80 \%$ of patients treated with autogenic bone graft and pedicle screw fixation ${ }^{10}$. We did pedicle screw rod fixation with liberal bony decompression in most of our cases with 
recurrence. Fibrous scar excision was not attempted in view of high incidence of dural tears. Liberal foraminotomies were supplemented with distraction to widen the foraminal space. Significant improvement was seen in almost all the cases.

\section{Conclusion}

Dissectomy is a strong predictor of early recurrence in patients with LCS. Excision of disc material leads to vertebral settling resulting in loss of height of foraminal space and recurrence of symptoms. Addition of TLIF after dissectomy prevents vertebral body settling and hence recurrence. In redo surgery excision of scar tissue often leads to major dural tears.Pedicle screw rod fixation with distraction is an excellent option in these cases as it leads to restoration of foraminal height.

\section{References}

[1]. Javalkar V, Cardenas R, Tawfik TA, et al. Reoperations after surgery for lumbar spinal stenosis. World Neurosurg. 2011;75 (56):737-742.

[2]. Jurícek M, Rehák L, Tisovský P, Horváth J. The effect of complications on the quality of life after surgery for lumbar spine degenerative disease. Acta Chir Orthop Traumatol Cech. 2010;77(2):112-117.

[3]. Weinstein JN, Tosteson TD, Lurie JD, et al. Surgical versus nonoperative treatment for lumbar spinal stenosis four-year results of the Spine Patient Outcomes Research Trial. Spine. 2010;35(14):1329-1338.

[4]. Wong C-B, Chen W-J, Chen L-H, Niu C-C, Lai P-L. Clinical outcomes of revision lumbar spinal surgery: 124 patients with a minimum of two years of follow-up. Chang Gung Med J.2002;25(3):175-182.

[5]. Atlas SJ, Keller RB, Wu YA, Deyo RA, Singer DE. Long-termoutcomes of surgical and nonsurgical management of lumbarspinal stenosis: 8 to 10 year results from the maine lumbarspine study. Spine. 2005;30(8):936-943.

[6]. Weinstein JN, Lurie JD, Tosteson TD, et al. Surgical compared with nonoperative treatment for lumbar degenerative spondylolisthesis. four-year results in the Spine Patient Outcomes Research Trial (SPORT) randomized and observational cohorts. J Bone Joint Surg Am. 2009;91(6):1295-1304.

[7]. Deyo RA, Martin BI, Kreuter W, et al. Revision surgery following operations for lumbar stenosis. J Bone Joint Surg Am. 2011;93(21):1979-1986.

[8]. Lai P-L, Chen L-H, Niu C-C, Fu T-S, Chen W-J. Relation between laminectomy and development of adjacent segment instability after lumbar fusion with pedicle fixation. Spine. 2004;29(22):2527-2532 [discussion 2532].

[9]. Altinkaya N, Yildirim T, Demir S, Alkan O, Sarica FB. Factors associated with the thickness of the ligamentum flavum: is ligamentum flavum thickening due to hypertrophy or buckling? Spine. 2011;36(16):E1093-E1097.

[10]. Chen L, Yang H, Tang T. Cage migration in spondylolisthesis treated with posterior lumbar interbody fusion using BAK cages. Spine. 2005;30(19):2171-2175. 